\title{
The Effect of the First Goal on Match Score in Football for the Home Field Advantage
}

\author{
Tuğbay Inan ${ }^{1}$, Beyza Cetin ${ }^{2}$, Ozge Gungorur ${ }^{3}$ \\ ${ }^{1}$ Faculty of Sports Sciences, Dokuz Eylul University, Izmir, Turkey \\ ${ }^{2}$ Faculty of, Science, Dokuz Eylul University, Izmir, Turkey \\ ${ }^{3}$ Institute of Health Sciences, Ege University, Izmir, Turkey \\ Correspondence: Tuğbay Inan, Faculty of Sports Sciences, Dokuz Eylul University, Izmir, Turkey.
}

Received: January 3, 2019

doi:10.11114/jets.v7i8.4331

\begin{abstract}
The interest in sports in the world is increasing in parallel with the developments in communication technology, . As a result of this increase, the number of researches conducted on sports industry and sport management has also showed an increase. The field of sport that attracts the most attention worldwide is football. Data analysis and statistical research are also included in football researches in this field of sports that intertwines with different fields of science such as football management, finance and sports industry. In addition to the econometric measurement methods on the related researches, the variables affecting the results of the competition and the statistical analyzes that determine the impact strength of these variables are more abundant in the literature. As a result of the competitions, the variables affecting the results of a competition attract the attention of football players, technical team, fans, media organizations and sports managers. When it comes to this study, it was planned to statistically determine the effect of the first goal in the European football leagues on the results of the match. From 5 countries in Europe Spain, France, Germany, England and Italy in particular, data belonging to the matches of the teams in which they were the home team between 2014-2017 seasons was used. A total of 5478 matches were analyzed in which 1140 matches were from Spain, 918 from Germany, 1140 from France, 1140 from England and 1140 from Italy. The relationship between the first goal and the match result was determined by the chi-square independence test. SPSS 24.0 package program was used in the analysis. The first goal independent variable in the study was examined and it was found that there was a relationship between the first goal and the match results $(\mathrm{p}<0.05)$. As far as results of the research data are concerned, it was found that there was a relationship between the first goal scored and the match results of the 5 major European football leagues when the teams were the home teams during the three seasons. Accordingly, the importance of the first goal was demonstrated by this study.
\end{abstract}

Keywords: European football, first goal, football, home field advantage, match score, soccer

\section{Introduction}

Sports concept has become an important part of human life as well as enabling social, cultural, political and economic gains. Today, sports is defined as a competitive physical activity with predetermined rules which is an interesting platform gathering millions of people including the sportsmen and audience. Sports feels charming for people because it offers the opportunity to socialize, and the possibility of any surprising goals in the competitions and matches. Observation of the performance parameters of the sportsmen during the race or match and pooling the statistical data and determination of the techniques and tactics of the sportsmen, in terms of team sports, are used to predict the match scores or to take precautions for future matches and to use previous data of team tactics. This type of analysis also guides the coach, the technical man and the sportsmen and develops them. The researchers show the total performance of a race or match, and prepares them for next matches. They sometimes develop several models to calculate possibilities and they work to predict scores in several branches. The literature shows that several methods are used to predict scores in several football branches.

The studies on sports economics in recent years have shown that several models have already been developed to determine the parameters affecting the match scores and the strength of these parameters. There are several parameters affecting the scores of sports competitions. Investigating the parameters by different categories constitutes the essense 
of the studies on sports economics and performance. The study performed by Simon Rottenberg on 1956 is accepted as the first step of studies on sports economics. Following this study, several studies were carried out to investigate the relationship between sports economy and football teams and the leagues' performances. In recent years, the studies have began to focus on combining sports economics, econometric measurement methods and team performances together.

Especially, since home field advantage is an important factor in determining the match score in football, several studies have been performed on this issue. No clear evidences for this advantage were determined in these long-term studies. This concept varies worldwide from country to country and even from league to league. However, the issue of home field advantage has been well known and studied since the first professional match on 1866. In a study performed by Pollard (1986), it was reported that there is a home field advantage especially in team sports.

In time, various methods have been developed by using econometrics, mathematics and statistics in order to predict the scores of sports matches. These methods are sold commercially online to people who train football teams, researchers and people who are interested in the scores of football matches. The created model systems evaluate the performances of the competitors and facilitate the analyses on winning races and taking precautions against competitors according to the predetermined criteria in team or individual sports (Leung et al, 2014).

The probability hypothesis in sports matches consists several methods based on previous competition data. Several studies have considered the matches in the football league as independent individual events, i.e. they suggest that the score of a match does not affect next matches. However, several investigators suggest that if the parameters used in probability hypothesis are correctly identified, the scores of the next matches could be predicted correctly and these scores might be useful as a tactic for team technical committee (Dixon et al, 1997), (Karlis et al, 2003).

Literature review showed that Hughes grouped performance analysis models under four categories: empirical models, dynamic systems, statistical techniques and artificial intelligence. (Hughes et al, 2007) and that sports matches have multiparameters affecting the match scores. The match scores are good indicators, but do not provide sufficient data for prediction. The parameters affecting match scores include the place where the match will be played, weather conditions, number of audience, the presence of any star football players, injured players, previous penalty cards and similar parameters. Therefore, researchers should analyze statistical data fastidiously and identify parameters correctly when developing prediction models. Therefore this study was planned to identify whether the first goal of the home team has effects on match scores in 5 big leagues in Europe and whether this parameter will be used in a future model.

\section{Method}

In the study, total of 5478 matches in 5 big football league in Europe were analyzed: 918 in Germany, 1440 in France, 1140 in England, 1140 in Spain and 1140 in Italy. Top leagues of countries, Bundesliga, Ligue 1, Premier Lig, La Liga and Serie A were watched in 2014-2017 to determine whether the home teams scored the first goal and won the match. Thus, data sets were created to get analyzed. The teams played 34 or 38 matches during the whole season. Data collected from the website www.mackolik.com was used for match analysis.

In the study, descriptive statistics were estimated as \% frequency for match scores of home team: won, draw or lost. Besides, the team (home or guest) scoring the first score for each league were listed in tables. On the other hand, the effect of this first goal on match were calculated separately for home and guest teams and defined as first goal advantage.

On the other hand, we used Pollard's procedure (1986) to determine the home field advantage. Accordingly, if the scores of a team as home is more than $50 \%$ of general scores of that team at the end of the league, it means there is a home field advantage for that team. If the rate is $\leq 50 \%$ there is not a home field advantage.

SPSS 24.0 statistical program was used to analyze data. Chi-square $\left(\chi^{2}\right)$ independence test was with $\mathrm{p}<0.05$ significance level was used to determine the relationship between the first goal and the match score, our statistical parameters.

\section{Results}

In this section, we presented the results from total of 5478 match scores in the important Europe football leagues which were analyzed.

Table 1 presented total number of matches, match scores (won, drew, lost) in each league, and the distribution of the team who scored the first goal. 
Table 1. Frequency and distribution of final outcome of a match according to scoring first home team in each league

\begin{tabular}{|c|c|c|c|c|c|}
\hline & & Lost & Win & Draw & Total \\
\hline \multirow{3}{*}{ 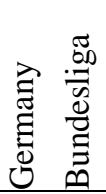 } & No first Goal & 213 & 62 & 130 & 405 \\
\hline & Scoring First & 54 & 361 & 98 & 513 \\
\hline & Total & 267 & 423 & 228 & 918 \\
\hline & No first Goal & 276 & 67 & 187 & 530 \\
\hline 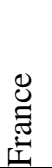 & Scoring First & 43 & 453 & 114 & 610 \\
\hline 晃 & Total & 319 & 520 & 301 & 1140 \\
\hline \multirow{4}{*}{ 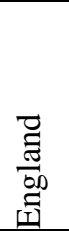 } & & Lost & Win & Draw & Total \\
\hline & No first Goal & 274 & 68 & 200 & 542 \\
\hline & Scoring First & 57 & 449 & 92 & 598 \\
\hline & Total & 331 & 517 & 292 & 1140 \\
\hline \multirow{3}{*}{$\begin{array}{l}\text { : } \\
\text { ڤี }\end{array}$} & No first Goal & 286 & 74 & 173 & 533 \\
\hline & Scoring First & 43 & 473 & 91 & 607 \\
\hline & Total & 329 & 547 & 264 & 1140 \\
\hline \multirow{3}{*}{ 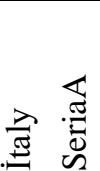 } & No first Goal & 308 & 70 & 164 & 542 \\
\hline & Scoring First & 58 & 449 & 90 & 597 \\
\hline & Total & 366 & 519 & 254 & 1139 \\
\hline
\end{tabular}

The study showed that home team scored the first goal in 513 matches among 918 matches in Germany Bundesliga, during 2014-2017 seasons. The home team scored the first goal in 610 among 1140 matches in Ligue1, 598 among 1140 in Premier Lig, 607 among 1140 in Laliga and 597 among 1139 in Seria A. Table 2 presented the frequency values by leagues.

Table 2. Frequency and distribution of first score of home teams

\begin{tabular}{ccccc}
\hline Bundesliga & Ligue 1 & Premier League & La Liga & Serie A \\
\hline$\% 56$ & $\% 54$ & $\% 52$ & $\% 53$ & $\% 52$ \\
\hline
\end{tabular}

Table 2 shows that the first goal possibility of home team is the highest for Bundesliga (56\%) and lowest for England Premier League and Italy SeriaA (52\%).

The other result of the study was associated with the study aim: to calculate the percentage of the home team winning when home team scored the first goal. The results showed that the home team scored the first goal in 513 matches in Bundesliga, and and the home team won in 361 of them. The home team won in 453 among 610 matches in Ligue1, 449 among 598 matches in Premier Lig, 473 among 607 matches in Laliga and 449 among 597 matches in Seria A. Frequency values of leagues were presented in table 3.

Table 3. Frequency and distribution of winning of home teams after scoring first goal by home team

\begin{tabular}{ccccc}
\hline Bundesliga & Ligue 1 & Premier League & La Liga & Serie A \\
\hline$\% 70$ & $\% 74$ & $\% 75$ & $\% 78$ & $\% 75$
\end{tabular}

The study results showed that; winning probability of home teams after scoring first goal by home team was highest in Spain Laliga (78\%), lowest in Bundesliga (70\%).

The other important result of the study was to determine at least $\geq 1$ score winning percentages after scoring first goal by home team. Accordingly, the winning team has 3 scores, the loser team has 0 scores at the end of the football match. If there is equality, each team has 1 score at the end of the football match. In Bundesliga, home team scored first goal in 513 matches and home team had $\geq 1$ scores in 459 among them. Home team had $\geq 1$ scores in 567 among 610 matches in Ligue1, 541 among 598 matches in Premier Lig, 564 among 607 matches in Laliga and 539 among 597 matches in Seria A. The frequency values of these data are presented in Table 4. 
Table 4. Frequency and distribution of at least $\geq 1$ score winning percentages of home teams after scoring first goal by home team

\begin{tabular}{ccccc}
\hline Bundesliga & Ligue 1 & Premier League & La Liga & Serie A \\
\hline$\% 89$ & $\% 93$ & $\% 90$ & $\% 93$ & $\% 90$
\end{tabular}

These results showed that the percentage of at least $\geq 1$ score winning after scoring first goal by home team was the highest in Spain Laliga and England Premier League (93\%), the lowest in Bundesliga (89\%). Again, these results showed that the losing and 0 scoring possibility of home teams after scoring first goal by home teams was as follows: in Bundesliga 11\%, in Ligue 1 7\%, in Premier league \%10, in Laliga 7\% and in SerieA 10\%. The other interesting result is the posibility of winning of home team unless the home team scored first goal: home team won $15 \%$ matches in Bundesliga, $13 \%$ in Ligue 1,13\% in Premier League, $14 \%$ in Laliga and 13\% in SerieA when the home team could not score first goal.

Besides, the other aim of the study was to determine the home field advantage in five big football leagues in Europe during 2014-2017 seasons. The frequency values of these data are presented in Table 5.

Table 5. Means of Home Field Advantage for Leagues

\begin{tabular}{ccccc}
\hline Bundesliga & Ligue 1 & Premier League & La Liga & Serie A \\
\hline$\% 59$ & $\% 59$ & $\% 58$ & $\% 60$ & $\% 58$
\end{tabular}

According to the 5478 match analyses of the important leagues in Europe, Table 5 showed that the home field advantage was the highest in Spain La Liga league (60\%), the lowest in Bundesliga and Ligue 1 (\%59), Premier League and Italian Seri A (\%58).

\section{Discussion}

Sports literature has shown that there are several studies investigating the numbers and types of the goals in a football match. However, there are limited studies investigating the direct effect of the first goal on match score. These limited studies were performed for match analysis. All of these studies suggested that the first goal in the football match is one of the important parameters affecting the match score.

The aim of the study was to determine the importance of the first goal of the home team in terms of the match score. The results of the study confirmed this concept. It is observed that the first goal by home team gives an advantage to this team in terms of the match score in 5 big leagues in Europe. The investigated football teams scored the first goal as home team in $53.4 \%$ of their matches. Even though this rate does not seem very advantageous for a football match (most of football scientists, trainers and coaches expect that the percentage of first goal by home team is high), the main advantage occurs following the first goal.

Armatas et al found in their study in 2009 that the teams who scored the first goal has the possibility to win the match by $70 \%$ to $75 \%$. (Armatas \& Yiannakos , $2009: 39-43$ ). Our study found that the home teams who scored the first goal wins the match by $70 \%$ to $78 \%$, and the median value was $74.4 \%$.

Courneya reported in their study in 1990 that the winning possibility of the team who scored the first goal in the match is high since this score gives a positive momentum as a psychologic factor (Courneya, 1990: 624-626). However, Schwartz and Barsky (1977) reported in their study that higher advantage of the home team may be associated with the quality of the home team (Schwartz \& Barsky, 1977: 641-661).

On the other hand, the other results of the study confirmed that the first goal in the 5 big Europe leagues is a very important parameter for the match score. The home teams only won the $13.6 \%$ of the matches unless they scored the first goal. Given that the home field advantage is generally over 50\% in Europe leagues, $13.6 \%$ is very low.

The other interesting study result was that the home team had at least 1 score in $91 \%$ of the matches if first goal was scored by home team. It means that the home team either won the match or equality was obtained after scoring first goal by home team. On the other hand, a positive significant correlation was found between the first goal and match scores when chi-square $\left(\chi^{2}\right)$ independence test was applied with $\mathrm{p}<0.05$ significance level by statistical results. These results were similar to the results by Tenga (2012) and Armatas et al (2009).

On the other hand, the study results for home field advantage are in line with several Europe studies on football leagues and home field advantage. The study results showed that the rate of home field advantage was $58.8 \%$ in 5 big Europe football leagues. Pollard \& Gómez (2014) reported that the average values were 59\% for Europe leagues.

The results showed that the first goal in football match is a robust parameter affecting match scores. However, this effect may vary by minutes of the first goal, the quality of the competitor, and the tactic of the competitor in the game. 
Even though this result seems important for football players, technical committee and club managers, it should be investigated in a larger data set in different leagues with different parameters including budget, star player profile in terms of attack styles of the first goals in order to increase study reliability.

\section{References}

Armatas, V., Yiannakos, A., Papadopoulou, S, et al (2009). Evaluation of Goals Scored in Top Ranking Soccer Matches: Greek "Superleague" 2006-07. Serbian Journal of Sports Sciences, (3), 39-43.

Carson, L., \& W.Joseph K (2014). Sports Data Mining: Predicting Results for the College Football Games. Procedia Computer Science, (35), 711-719. https://doi.org/10.1016/j.procs.2014.08.153

Courneya, K. S. (1990). Importance of Game Location and Scoring First in College Baseball. Perceptual and Motor Skills, (71), 624-626. https://doi.org/10.2466/PMS.71.6.624-626

Dixon, M. J., \& Coles, S. G. (1997). Modelling Association Football Scores and Inefficiencies in the Football Betting Market, Journal of the Royal Statistical Society, Series C, (46), 265-280. https://doi.org/10.1111/1467-9876.00065

Hughes, M., \& Franks, I. (2007). The Essentials of Performance Analysis:An Introduction, New York, NY Routluge. https://doi.org/10.4324/9780203938065

Karlis, D., \& Tzoufras, N. L. (2003). Analysis of Sports Data by Using Bivariate Poisson models, Journal of the Royal Statistical Society, Series D(52), 381-393. https://doi.org/10.1111/1467-9884.00366

Leung, C., \& W. Joseph, K. (2014). Sports Data Mining: Predicting Results for the College Football Games. Procedia Computer Science, (35), 710-719. https://doi.org/10.1016/j.procs.2014.08.153

Pollard, R. (1986). Home Advantage in Soccer:A Retrospective Analysis, Journal of Sports Sciences, (3), 237-248. https://doi.org/10.1080/02640418608732122

Pollard, R., \& Gómez, M. A. (2014). Comparison of home advantage in men's and women's football leagues in Europe. European Journal of Sport Science, (14), 77-83. https://doi.org/10.1080/17461391.2011.651490

Rottenberg, S. (1956). The Baseball Player's Labor Market, Journal of Political Economy, (64), 242-258. https://doi.org/10.1086/257790

Schwartz, B., \& Barsky, S. F. (1977). The Home Advantage. Social Forces, (55), 641-661. https://doi.org/10.1093/sf/55.3.641

Tenga, A. (2012). First Goal and Home Advantage at Different Levels of Play in Professional Soccer. World Congress of Performance Analysis of Sport IX, 47-51.

\section{Copyrights}

Copyright for this article is retained by the author(s), with first publication rights granted to the journal.

This is an open-access article distributed under the terms and conditions of the Creative Commons Attribution license which permits unrestricted use, distribution, and reproduction in any medium, provided the original work is properly cited. 